\title{
Treating multiple sclerosis with generic cladribine
}

\author{
Alvarez-Gonzalez $\mathrm{C}^{1,2}$, Allen-Philbey $\mathrm{K}^{2}$, Mathews $J^{3}$, Espasandin $\mathrm{M}^{2}$, Turner $\mathrm{BP}^{1,2}$, Gnanapavan $\mathrm{S}^{1,2}$, Marta $\mathrm{M}^{1,2}$
} Giovannoni $\mathrm{G}^{1,2}$, Baker $\mathrm{D}^{1}$, Schmierer $\mathrm{K}^{1,2}$

${ }^{1}$ Blizard Institute (Neuroscience), Queen Mary University of London, London, UK; ${ }^{2}$ Barts Health NHS Trust, Emergency Care \& Acute Medicine Neuroscience Clinical Academic Group, London, UK; ${ }^{3}$ Barts Health NHS Trust, Neurology and Medicines Safety, London, UK.

Contact: 4 Newark Street, London, E1 2AT, UK. cesar.gonzalez@qmul.ac.uk k.schmierer@qmul.ac.uk

@NeuroWandererMx / @KlausSchmierer / @BartsMSBlog

\section{Introduction}

Phase II \& III trial evidence suggests Cladribine (CLAD), a purine analogue licensed for treatment of people with hairy cell leukaemia, is also an effective, safe and convenient disease modifying therapy (DMT) for people with multiple sclerosis (pwMS) ${ }^{1-5}$. Cladribine exerts its effect through selective long-term suppression of lymphocytes ${ }^{6}$ and reduction of pro-inflammatory cytokines and chemokines ${ }^{7}$ whilst leaving other cell types relatively unaffected. Here we report our experience with CLAD in pwMS using a dosing scheme that adapts to individual lymphocyte level

\section{Methods}

This study is registered with the Clinical Effectiveness Unit of Barts Health NHS Trust (\#7142). CLAD was offered to pwMS with clinical and/or MRI disease activity who either (i) fulfilled NHS England (NHSE) criteria for licensed DMT, however chose CLAD instead, or (ii) as a compassionate treatment in pwMS not eligible for DMT according to NHSE criteria ${ }^{8}$. Written informed consent was obtained from patients after they were fully informed about the off-license status of CLADas a DMT for pwMS. A comprehensive safety check list (fig 1) was applied prior to treatment initiation. Treatment consisted of up to two annual cycles of subcutaneous Cladribine (Litak®). During each cycle $10 \mathrm{mg}$ Cladribine was given on up to 7 days. The number of injections administered was adjusted to individual lymphocyte count to avoid lymphopenia below $0.5 \times 109 / \mathrm{L}$ (see fig 1 for details of the dosing scheme). One way ANOVA was used to analyse differences in white cell counts, and any reported adverse events recorded. Results after a mean follow-up of 4 months are reported.

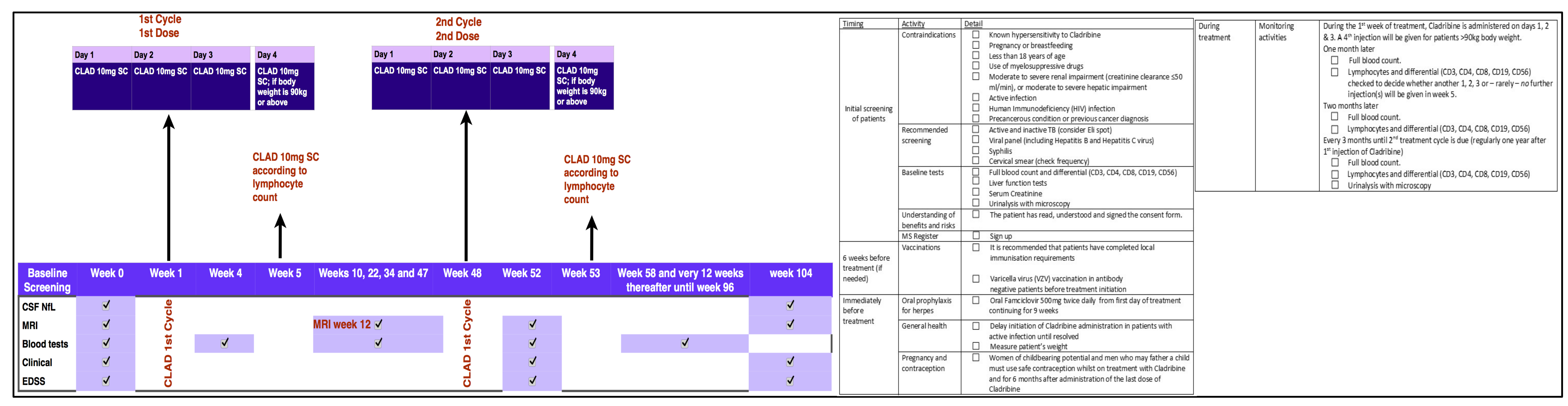

Figure 1. Dosing scheme adjusted to individual lymphocyte count and safety check list

Results

Twenty five pwMS (14 women and 11 men) had a first treatment cycle of CLAD. Median age was $42(31-60)$ years, and median EDSS 5 (1.5-8). No evidence of prior infectionor other significant risk factors were identified accordingto our check-list. Nine patients were treatment naïve and chose CLAD as their first DMT. One patient had taken part in CLARITY $^{2}$, and resumed disease activity five years after a Gd-enhancing lesion was last detected on MRI. Five patients were not eligible for NHSE approved DMT and received Cladribine on a compassionate basis. Twelve pwMS switched from other DMTs to Cladribine due to side effects and/or an individual risk-benefit assessment. Three patients developed adverse events: Two had mild skin infections (rash on the hip, and astye); one patient had transient abdominal pain leading A\&E review with no cause identified and spontaneous remission. One 39-year old man had a myocardical infarct (MI) four weeks after the 6th Cladribine injection. Vascular risk factors in this patient included smoking and hypercholesterolemia, and his MI therefore not deemed treatment-related. No clinical disease activity was observed in any of the pwMS included within the follow-up period. Total lymphocyte count dropped from a mean of 1.82 (range: $0.9-3.4 \times 109$ ) at baseline to $1.34(0.7-3)$ at 4 weeks, and to $1.18(0.6-1.6)$ at 8 weeks, $p=0.014$. The lowest lymphocyte count observed was $0.6 \times 109$. Other white cells remained within normal range.

Disease activity and the reasons of Cladribine administration

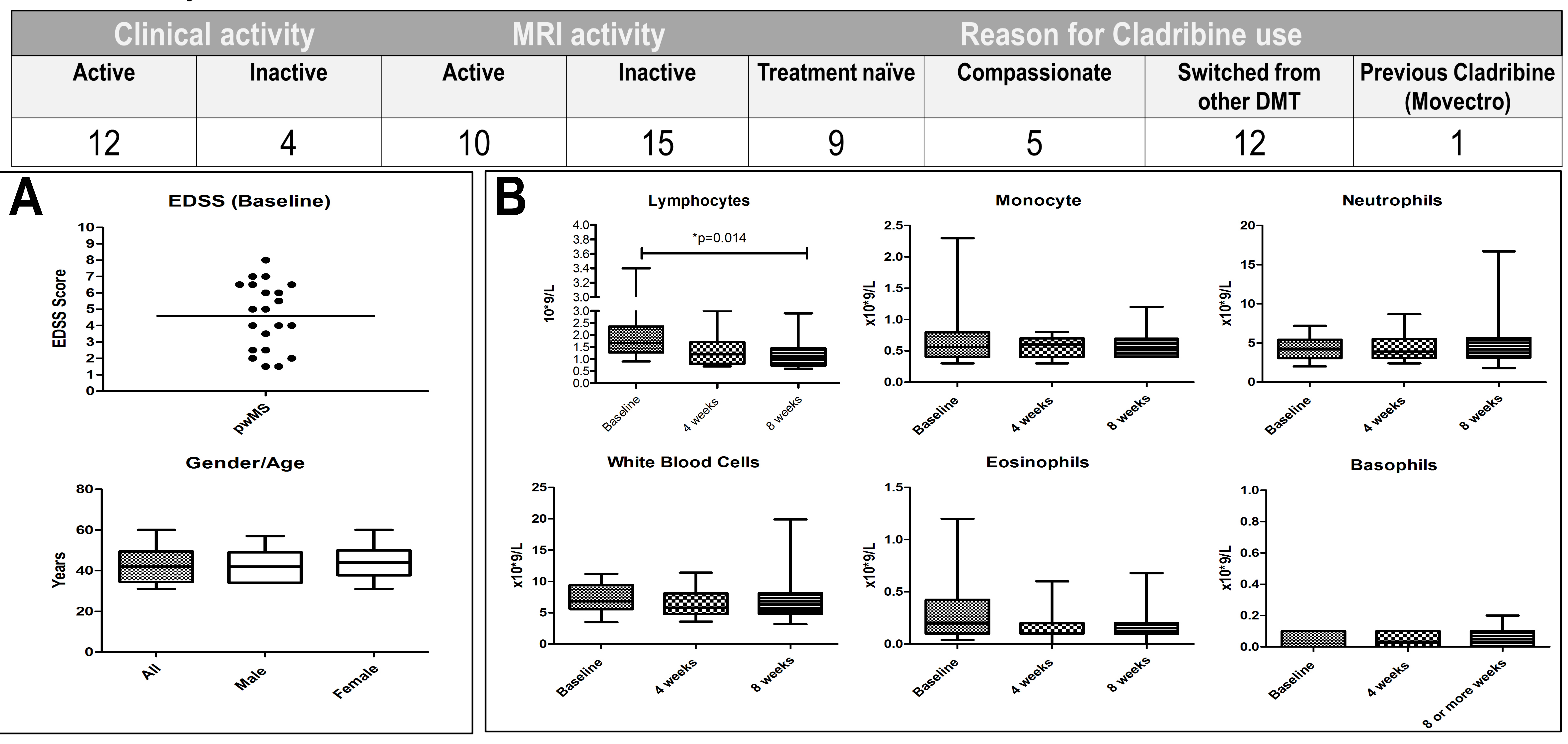

A) Demographic data of pwMS

Conclusion

Generic subcutaneous CLAD was generally well tolerated. Our novel personalised dosing scheme adapted to individual lymphocyte count led to controlled depletion of lymphocytes in all pwMS leaving other white cells unaffected.
To date there no clinical evidence of disease activity has emerged and further follow-up is underway to evaluate the long term effects of CLAD in this cohort. Though not currently licensed as a DMT for pwMS, CLAD deserves consideration as an off-label alternative. 